\title{
Immunogenicity of a Quadrivalent Human Papillomavirus Vaccine When Co-Administered with Tetanus-Reduced Diphtheria-Acellular Pertussis and Quadrivalent Meningococcal Conjugate Vaccines in Healthy Adolescents: Results from a Randomized, Observer-Blind, Controlled Trial
}

Yan Miao · Thembile Mzolo • Michele Pellegrini (D)

Received: April 18, 2019 / Published online: August 3, 2019

(C) The Author(s) 2019

\section{ABSTRACT}

Introduction: Vaccines against human papillomavirus (HPV), tetanus, diphtheria, pertussis (Tdap) and Neisseria meningitidis are widely recommended in adolescents. A phase- 4 observerblind study was performed to investigate the impact of concomitant administration of a quadrivalent HPV (HPV4) and Tdap vaccine with a quadrivalent meningococcal $\mathrm{CRM}_{197^{-}}$ conjugate vaccine (MenACWY-CRM) in terms of immunogenicity against the different vaccine antigens and overall safety profile. Previous results showed that concomitant administration of the three vaccines did not impact the immunogenicity of Tdap and MenACWY, or safety. This article presents recently released HPV immunogenicity results.

Methods: Healthy adolescents aged 11-18 years (801) were randomized to receive either HPV4 + Tdap + MenACWY or HPV4 + Tdap + Placebo and two additional doses of HPV4 at 2 and 6 months after the first dose.

Enhanced Digital Features To view enhanced digital features for this article go to https://doi.org/10.6084/ m9.figshare.8984045.

Y. Miao · T. Mzolo

GSK, Amsterdam, The Netherlands

M. Pellegrini $(\bowtie)$

GSK, Siena, Italy

e-mail: michele.x.pellegrini@gsk.com
Antibody responses to HPV types (HPV-6, -11, -16 and -18) were assessed at baseline and at 1 month post-full vaccination.

Results: Post-third HPV4 dose, non-inferiority of immune responses to HPV4 + Tdap + MenACWY vs. HPV4 + Tdap + Placebo was demonstrated; the lower limits of two-sided $95 \%$ CIs of the between-group differences in seroconversion rates were $>-5 \%$ (non-inferiority margin) against each HPV type tested. Seroconversion rates ranged between 98.0\% (HPV-6) and 99.7\% (HPV-11 and HPV-18) in group HPV4 + Tdap + MenACWY and from 99.0\% (HPV-11 and HPV-16) to 99.7\% (HPV-6 and HPV-18) in group HPV4 + Tdap + Placebo. Conclusion: Overall, these data support the concomitant administration of HPV4, Tdap and MenACWY-CRM in adolescents.

Funding: Novartis Vaccines and Diagnostics Inc., now part of the GSK group of companies. Trial Registration: ClinicalTrials.gov identifier, NCT01424644.

Keywords: Adolescents; Co-administration; Human papillomavirus vaccine; Quadrivalent meningococcal conjugate vaccine; Tetanusdiphtheria-acellular pertussis vaccine

\section{INTRODUCTION}

When considering current epidemiologic data and immunization practices, human 
papillomavirus (HPV), meningococcal and tetanus-reduced diphtheria-acellular pertussis (Tdap) vaccines are among those generally recommended to be administered in adolescence $[1,2]$. Whenever possible, concomitant administration of different vaccines, usually at different injection sites, is adopted to try to maximize the opportunities for improving compliance with vaccination schedules and community protection, as long as there is no immunologic interference among the co-administered vaccines.

A phase-4, clinical study was conducted in the USA and Italy to demonstrate non-inferiority of immune responses of a Tdap vaccine (Boostrix, GSK) and quadrivalent HPV vaccine (HPV4; Gardasil, Merck \& Co., Inc.) when administered concomitantly with a quadrivalent meningococcal $\mathrm{CRM}_{197}$-conjugate vaccine (MenACWY-CRM; Menveo, GSK) or placebo in healthy adolescents aged 11-18 years. As immunogenicity results related to Tdap and MenACWY-CRM reference antigens and safety data were available, they were previously published [3]. In this article, the latest available analyses pertaining to the assessment of the HPV4 immunogenicity, when the vaccine was administered concomitantly with Tdap and MenACWY-CRM or placebo, are presented.

\section{METHODS}

This was a phase- 4 , multicenter, randomized, observer-blind, controlled trial (NCT01424644), conducted across 20 sites in the US and Italy between September 2011 and December 2012, in which healthy adolescents $11-18$ years of age were randomized $1: 1$ to concomitantly receive one dose each of HPV4, Tdap and MenACWYCRM (group HPV4 + Tdap + MenACWY) or one dose each of HPV4, Tdap and placebo (group HPV 4 + Tdap + Placebo). All participants received two additional doses of HPV4, administered 2 and 6 months after the first dose. Further details on the study design, randomization, blinding, interventions and sample size calculations have been previously published [3].
Eligible study participants included those who had given their written consent/assent and who had received childhood vaccinations against diphtheria, tetanus and pertussis, as per local regulations, but had not yet received any adolescent dose of vaccines against diphtheria, tetanus or pertussis. Eligible female individuals were required to have a negative urine pregnancy test and, if sexually active, to be willing to adopt appropriate birth control measures during the study period. Main exclusion criteria were: history of anaphylaxis/serious reactions to any component of the study vaccines; previous disease (confirmed or suspected) caused by $N$. meningitidis; receipt of any investigational or licensed vaccines containing meningococcal antigens or a prior HPV vaccination; history of any serious acute, chronic or progressive disease, or of an impaired immune system. A more detailed list of inclusion and exclusion criteria has been published elsewhere [3].

The study was conducted in accordance with the Declaration of Helsinki and the Good Clinical Practice guidelines. Prior to enrollment, approval of the study protocol and protocolrelated documents was obtained from the relevant Independent Ethics Committees. Informed consent was obtained from all individual participants included in the study or, for those < 18 years of age, from their parents or legal guardians.

Here, we describe the results of the co-primary objective of the study linked to HPV4 immunogenicity, which was to demonstrate non-inferiority of the immune responses induced by HPV4 given concomitantly with Tdap and MenACWY-CRM compared with those elicited by HPV4 co-administered with Tdap and placebo, 1 month after the third HPV4 dose. Non-inferiority was demonstrated if the lower limits of the two-sided 95\% confidence intervals (CIs) on the between-group differences (HPV4 + Tdap + MenACWY group minus HPV4 + Tdap + Placebo group) in seroconversion (SC) rates were $>-5 \%$ for each of the HPV types 6 (HPV-6), HPV-11, HPV-16 and HPV-18.

Immune responses to HPV4 vaccination were assessed using blood samples collected at baseline, before the first HPV4 dose and 
1 month after the third HPV4 dose. Serum antibody levels against the four HPV types were measured by means of a validated competitive Luminex immunoassay used for the evaluation of immune responses to HPV4 during its clinical development program $[4,5]$.

SC rates were calculated at 1 month after the third HPV4 dose for each study group and HPV type as the percentage of participants with antibody titers greater or equal to a HPV typespecific cutoff and were seronegative at baseline (i.e., had antibody titers lower than the cutoff value). The pre-established cutoff value was 16 milliMerck units (mMU)/ml for HPV-11, 20 $\mathrm{mMU} / \mathrm{ml}$ for HPV-6 and HPV-16, and $24 \mathrm{mMU} /$ $\mathrm{ml}$ for HPV-18. The associated two-sided 95\% CIs for SC rates in each group were calculated using the Clopper-Pearson method [6], and those for the between-group differences in SC rates were computed using the Miettinen-Nurminen method [7].

Geometric mean antibody titers (GMTs) and geometric mean ratios (GMRs; post-vaccination over baseline antibody levels) were also calculated by study group, by exponentiating ( base $_{10}$ ) the corresponding least square means of the $\log _{10}$-transformed titers. The associated $95 \%$ CIs were derived using the analysis of variance (ANOVA), with study group and center in the model.

The main immunogenicity analysis population was the HPV per-protocol (HPV-PP) set, which included all participants without major protocol deviations, who correctly received all vaccine doses and for whom evaluable serum samples were available for at least one HPV type at both baseline and at 1 month post-third dose, with vaccine injection and blood draw occurring within the protocol-required windows.

Assuming a 10\% dropout rate, approximately 800 individuals were planned to be enrolled in the study (400 per vaccine group) to obtain a sample size of 360 evaluable participants in each group. The power to demonstrate non-inferiority for each HPV type was $\geq 99.8 \%$, using a non-inferiority margin of $-5 \%$. More details on the study sample size calculation and overall study power have been reported previously [3].

\section{RESULTS}

Of the 801 participants enrolled in this study (247 in Italy and 554 in the US), 634 were included in the HPV-PP set: 309 in the HPV4 + Tdap + MenACWY group and 325 in the HPV 4 + Tdap + Placebo group. The main reasons for exclusion were administration of prohibited vaccination and non-compliance with vaccination or blood draw schedule. The demographic characteristics of participants in the HPV-PP set were overall balanced between the study groups. The mean age of the participants was $11.9( \pm 1.6)$ years, and $38 \%$ were females (Table 1).

One month after the third HPV4 dose, SC rates ranged between 98.0\% (HPV-6) and 99.7\% (HPV-11 and HPV-18) in HPV4 + Tdap + MenACWY group and between 99.0\% (HPV-11 and HPV-16) and 99.7\% (HPV-6 and HPV-18) in the HPV4 + Tdap + Placebo group (Fig. 1a). Differences between the SC rates in the HPV4 + Tdap + MenACWY group and HPV 4 + Tdap + Placebo group were - 1.0\% (95\% CIs - 3.5\% to 0.3\%) for HPV-6, 0.3\% (95\% CIs $-1.2 \%$ to $1.9 \%)$ for HPV-11, $1.0 \%$ (95\% CIs $-1.2 \%$ to $2.6 \%$ ) for HPV-16 and $0.0 \%$ (95\% CIs $-1.5 \%$ to $1.4 \%)$ for HPV-18, with the lower limit of the $95 \%$ CIs being $>-5 \%$ for each tested HPV type (Fig. 1b). Therefore, immune responses to HPV4 given concomitantly with Tdap and MenACWY-CRM were shown to be non-inferior to the responses of HPV4 when coadministered with Tdap and placebo.

At baseline, GMTs were similar between the two groups for each HPV type. One month postthird HPV4 dose, robust antibody responses were observed in both groups for all HPV types (Fig. 1c). GMTs ranged between 1012.60 (HPV6) and 6293.21 (HPV-16) in the HPV4+ Tdap + MenACWY group and between 1084.13 (HVP-6) and 7221.71 (HPV-16). GMTs were comparable between groups, with overlapping 95\% CIs, when analyzed by HPV type (Fig. 1c).

One month post-third HPV4 dose, GMRs ranged between 173.85 (HPV-6) and 1111.67 (HPV-16) in the HPV4 + Tdap + MenACWY group and between 191.15 (HPV-6) and 1277.5 (HPV-16) in the HPV4 + Tdap + Placebo group 
Table 1 Characteristics of study participants at baseline (HPV per-protocol population)

\begin{tabular}{llll}
\hline & $\begin{array}{l}\text { Group } \\
\text { HPV4 + Tdap + MenACWY }\end{array}$ & $\begin{array}{l}\text { Group } \\
\text { HPV4 + Tdap + Placebo }\end{array}$ & Total \\
\hline $\mathrm{N}$ & 309 & 325 & 634 \\
Mean age $( \pm \mathrm{SD})$, years & $11.9 \pm 1.63$ & $11.8 \pm 1.48$ & $11.9 \pm 1.56$ \\
Female, $n(\%)$ & $124(40 \%)$ & $116(36 \%)$ & $240(38 \%)$ \\
Ethnicity, $n(\%)$ & & $6(2 \%)$ & $9(1 \%)$ \\
Asian & $3(1 \%)$ & $26(8 \%)$ & $51(8 \%)$ \\
Black or African & $25(8 \%)$ & $260(80 \%)$ & $513(81 \%)$ \\
Caucasian & $253(82 \%)$ & $1(<1 \%)$ & $2(<1 \%)$ \\
American Indian/Alaskan Native & $1(<1 \%)$ & $2(1 \%)$ & $2(<1 \%)$ \\
Pacific/Hawaii & 0 & $30(9 \%)$ & $57(9 \%)$ \\
Other & $27(9 \%)$ & $49.92 \pm 15.08$ & $50.07 \pm 14.94$ \\
Mean weight $( \pm \mathrm{SD}), \mathrm{kg}$ & $50.22 \pm 14.81$ & $153.3 \pm 11.09$ & $153.2 \pm 11.39$ \\
Mean height $( \pm \mathrm{SD}), \mathrm{cm}$ & $153.1 \pm 11.72$ & $21 \pm 4.82$ & $21.1 \pm 4.75$ \\
Mean body mass index $( \pm \mathrm{SD})$, & $21.2 \pm 4.68$ & & \\
kg/m & & & \\
\hline
\end{tabular}

$H P V$ human papillomavirus, HPV4 quadrivalent HPV vaccine, Tdap tetanus-diphtheria-acellular pertussis vaccine, MenACWY quadrivalent meningococcal conjugate vaccine, $N$ number of participants in each group, $S D$ standard deviation, $n(\%)$ number (percentage) of participants in each category

(Fig. 1d). Overall, GMTs and GMRs were higher against HPV-16 and lower against HPV-6, irrespective of the study group.

\section{DISCUSSION}

The analyses on the immunogenicity of the HPV4 vaccine administered concomitantly with Tdap and MenACWY-CRM or placebo confirmed previously reported data, supporting the co-administration of the three vaccines with no impact on the immunogenicity of each vaccine antigen [8] or safety [3, 8]. Overall, the mean age of the population enrolled in this study was 11.9 years, which was in line with the age range generally recommended for HPV routine vaccination (11-12 years) [1, 2].

When HPV4 was co-administered with Tdap and the MenACWY-CRM, SC rates for all tested HPV types were $\geq 98 \%$ and were non-inferior to those observed when placebo was given instead of MenACWY-CRM. Post-vaccination GMTs and ratios were comparable between study groups, when analyzed by HPV type, with higher titers observed against HPV-16 and lower against HPV-6. In the same study, as previously reported [3], 1 month post-vaccination, 95\% and $99 \%$ of participants in the MenACWY-CRM group had seroprotective antibody levels $\geq 1.0 \mathrm{IU} / \mathrm{ml}$ ) against the diphtheria and tetanus toxoids, respectively, compared with $82 \%$ and $98 \%$ among the placebo recipients. Ratios of geometric mean concentrations of antibodies against pertussis antigens, pertussis toxin, filamentous hemagglutinin and pertactin for the MenACWY-CRM group versus placebo were $1.01,0.84$ and 0.82 , respectively. Overall, non-inferiority criteria for immunologic responses against all Tdap antigens were met. In addition, administration of a single dose of MenACWY-CRM elicited robust antibody responses against the four meningococcal 

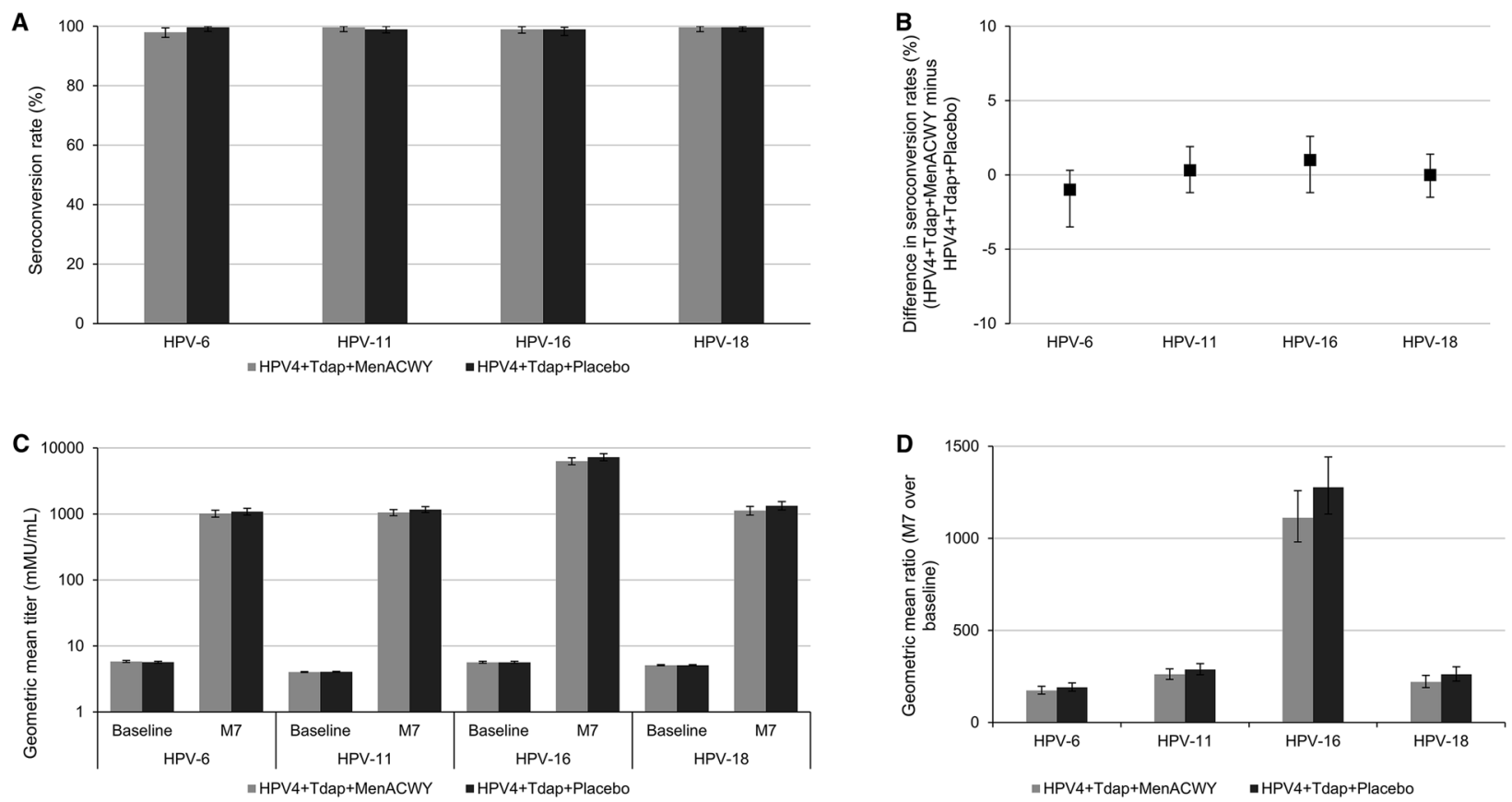

Fig. 1 Summary of immune responses to HPV types: seroconversion rates (a) and between-group difference in seroconversion rates (b), geometric mean titers (c) and geometric mean ratios (d). $H P V$ human papillomavirus, HPV4 quadrivalent HPV vaccine, Tdap tetanus-

serogroups, with $77 \%, 84 \%, 95 \%$ and $86 \%$ of participants showing human complement serum bactericidal antibody titers $\geq 8$ against serogroups A, C, W and $\mathrm{Y}$, respectively [3].

Previous studies have assessed the co-administration of a different quadrivalent meningococcal conjugate vaccine with HPV and Tdap vaccines. A study in 10-17-year-old adolescents showed non-inferior immune responses to HPV4 when a quadrivalent diphtheria-toxoid conjugate vaccine (Menactra, Sanofi Pasteur Inc., France) and a Tdap vaccine (Adacel, Sanofi Pasteur Inc., France) were given concomitantly with the first HPV4 dose or 1 month later [9]. Another trial using the same vaccination schedule showed similar results in adolescents aged 11-15 years with a nonavalent HPV vaccine (Gardasil-9, Merck \& Co., Inc., USA) instead of HPV4 [10].

Another study assessed HPV4, Tdap and MenACWY-CRM, but compared concomitant with sequential administration of the three vaccines [8]. Responses to HPV4 were robust diphtheria-acellular pertussis vaccine, MenACWY quadrivalent meningococcal conjugate vaccine, $m M U$ milliMerck units, $M 7$ month 7 ( 1 month post-third HPV4 dose). Error bars represent $95 \%$ confidence intervals

and comparable among vaccination schedules [8], showing that concomitant administration of the three vaccines does not impact the individual immune responses to HPV types, consistent with observations in the current study.

When comparing safety data from participants exposed to the HPV4 vaccine concomitantly administered with Tdap and MenACWYCRM with those recorded in participants exposed to HPV4, Tdap and placebo, similar percentages of participants in the current study reported any adverse events [51\% versus 50\%, respectively, with injection site pain (7\% versus $6 \%$ ) and pharyngitis (6\% versus $5 \%$ ) being the most frequent ones] [3]. A similar incidence was observed in the two groups for medically attended adverse events $(20 \%$ and $24 \%$, respectively, none judged as related to the study vaccines) and new onset of chronic disease (6\% and $7 \%$, respectively), with the most frequently reported chronic conditions being seasonal allergies and minor dermatology conditions, such as acne and atopic dermatitis, consistent 
with expected patterns for this age group. Overall, seven participants (four in the HPV + Tdap + MenACWY group and three in the HPV + Tdap + Placebo group) reported nine serious adverse events during the entire study period; none of these were considered to be related to study vaccines [3].

Potential limitations of the study included the absence of a HPV4 + MenACWY + Placebo arm and the fact that the results have been mainly generated in individuals aged 11-12 years (which is the age generally recommended for HPV vaccination) and not in older adolescents.

\section{CONCLUSION}

Immunogenicity results for HPV types, together with the previously evaluated immune responses to the meningococcal, tetanus, diphtheria and pertussis antigens and safety data, further reinforce the feasibility of HPV4, Tdap and MenACWY-CRM concomitant administration in adolescents.

\section{ACKNOWLEDGEMENTS}

The authors thank the participants and investigators of the study.

Funding. This study was sponsored by Novartis Vaccines and Diagnostics Inc., now part of the GSK group of companies. GlaxoSmithKline Biologicals SA covered all costs associated with the development and publishing of the manuscript. All authors had full access to all of the data in this study and take complete responsibility for the integrity of the data and accuracy of the data analysis.

Medical Writing, Editorial and Other Assistance. The authors thank David Alexanderian (ex-Novartis Pharma), Noemi Giglioli (Novartis Pharma), William Johnston (Birmingham Pediatrics, Birmingham, AL, USA), Ellen Ypma (GSK), Jacqueline Mobius (GSK) and Susanne Pittl (GSK) for their contribution to the study conduct. The authors would also like to thank Modis platform for editorial assistance and manuscript coordination, Petronela Petrar (Modis c/o GSK) for providing medical writing services and Pauline De Berdt (Modis c/o GSK) for publication management. This assistance was funded by GlaxoSmithKline Biologicals SA.

Authorship. All named authors meet the International Committee of Medical Journal Editors (ICMJE) criteria for authorship for this article, take responsibility for the integrity of the work as a whole, and have given their approval for this version to be published. All authors contributed equally to the study.

Disclosures. Yan Miao is an employee of the GSK group of companies. Thembile Mzolo is an employee of the GSK group of companies. Michele Pellegrini is an employee of the GSK group of companies and holds shares in the GSK group of companies.

Compliance with Ethics Guidelines. All procedures performed in studies involving human participants were in accordance with the ethical standards of the institutional and/or national research committee and with the 1964 Helsinki Declaration and its later amendments or comparable ethical standards. Approval of the study protocol and protocol-related documents was obtained prior to enrollment from the following institutional review board/ethics committees: Schulman Associates Institutional Review Board Inc., Cincinnati, OH, US; Independent Ethics Committee of A.S.L. di Taranto, Taranto, Italy; Independent Ethics Committee of IRCCS Azienda Ospedaliera Universitaria San Martino, Genova, Italy; Independent Ethics Committee of Azienda Ospedaliero-Universitaria Maggiore della Carità di Novara, Novara, Italy. Informed consent was obtained from all individual participants included in the study or, for those under 18 years of age, from their parents or legal guardians.

Data Availability. The product that is studied in this clinical study, together with the rights to the data and results generated, has been transferred to GSK by Novartis. For interventional studies that evaluate GSK medicines, 
anonymized patient-level data are made available to independent researchers, subject to review by an independent panel, at http://www. clinicalstudydatarequest.com within 6 months of publication. To protect the privacy of patients and individuals involved in our studies, GSK does not publicly disclose patient-level data. Patient level data for this study will be made available on http://www. clinicalstudydatarequest.com upon request, subject to any pre-existing rights and obligations and/or consents required under the relevant agreements governing or related to these studies. Menveo and Boostrix are trademarks owned by the GSK group of companies. Gardasil and Gardasil-9 are trademarks owned by Merck \& Co., Inc. Menactra and Adacel are trademarks owned by Sanofi Pasteur Inc.

Open Access. This article is distributed under the terms of the Creative Commons Attribution-NonCommercial 4.0 International License (http://creativecommons.org/licenses/ by-nc/4.0/), which permits any noncommercial use, distribution, and reproduction in any medium, provided you give appropriate credit to the original author(s) and the source, provide a link to the Creative Commons license, and indicate if changes were made.

\section{REFERENCES}

1. Centers for Disease Control and Prevention. Recommended immunization schedule for children and adolescents aged 18 years or younger, United States. https://www.cdc.gov/vaccines/schedules/ downloads/child/0-18yrs-child-combined-schedule. pdf. Accessed 11 Apr 2019.

2. European Centre for Disease Prevention and Control. Vaccine scheduler. https://vaccine-schedule. ecdc.europa.eu. Accessed 11 Apr 2019.
3. Gasparini R, Johnston W, Conversano M, Garscadden A, Alexanderian D. Immunogenicity and safety of combined tetanus, reduced diphtheria, acellular pertussis vaccine when co-administered with quadrivalent meningococcal conjugate and human papillomavirus vaccines in healthy adolescents. J Vaccines Vaccin. 2014;5(3):231.

4. Dias D, Van Doren J, Schlottmann S, Kelly S, Puchalski D, Ruiz W, et al. Optimization and validation of a multiplexed luminex assay to quantify antibodies to neutralizing epitopes on human papillomaviruses $6,11,16$, and 18. Clin Diagn Lab Immunol. 2005;12(8):959-69.

5. Opalka D, Lachman CE, MacMullen SA, Jansen KU, Smith JF, Chirmule N, et al. Simultaneous quantitation of antibodies to neutralizing epitopes on virus-like particles for human papillomavirus types $6,11,16$, and 18 by a multiplexed luminex assay. Clin Diagn Lab Immunol. 2003;10(1):108-15.

6. Clopper C, Pearson S. The use of confidence or fiducial limits illustrated in the case of the binomial. Biometrika. 1934;26(4):404-13.

7. Miettinen O, Nurminen M. Comparative analysis of two rates. Stat Med. 1985;4(2):213-26.

8. Arguedas A, Soley C, Loaiza C, Rincon G, Guevara S, Perez A, et al. Safety and immunogenicity of one dose of MenACWY-CRM, an investigational quadrivalent meningococcal glycoconjugate vaccine, when administered to adolescents concomitantly or sequentially with Tdap and HPV vaccines. Vaccine. 2010;28(18):3171-9.

9. Reisinger KS, Block SL, Collins-Ogle M, Marchant C, Catlett M, Radley D, et al. Safety, tolerability, and immunogenicity of Gardasil given concomitantly with Menactra and Adacel. Pediatrics. 2010;125(6):1142-51.

10. Schilling A, Parra MM, Gutierrez M, Restrepo J, Ucros S, Herrera $T$, et al. Coadministration of a 9-valent human papillomavirus vaccine with meningococcal and Tdap vaccines. Pediatrics. 2015;136(3):e563-72. 